Proof. We have only to prove: if $X \subset P$ is discrete and is not a $Q$-space, then $P$ cannot be a $Q$-space. Put $S=P-(\bar{X}-X)$. Then $X$ is a closed subset of $S$, hence, by the above theorem, $S$ is not a $Q$-space. Since $P$ is metrisable, $S \in \boldsymbol{F}_{\sigma}(P), S=\sum_{n=1}^{\infty} S_{n}, S_{n} \in \boldsymbol{F}(P)$. Lemma 7 implies that some $S_{n}$ is not a $Q$-space; therefore, by Proposition $4, P$ is not a $Q$-space.

Remark. It is easy to show that a discrete space is a $Q$-space if and only if it does not admit of a non-reducible two-valued Borel measure, that is if its power has two-valued measure zero. Therefore, Theorem 3 may be given the following equivalent form: A fully normal space $P$ is a $Q$-space if and only if the power of any closed discrete subset of $P$ has two-valued measure zero.

\title{
References.
}

[1] J. Dieudonné, Une généralisation des espaces compacts, J. Math. Pures et Appliquées (9) 23 (1944), pp. 65-76.

[2] E. Hewitt, Rings of Continuous Functions, I, Trans. Amer. Math. Soc. 64 (1948), pp. 45-99.

[2a] E. Hewitt, Linear functionals on spaces of continuous functions, Fund: Math. 37 (1950), pp. 161-189.

[3] C. Kuratowski, Fopologie I, 2-me éd., Warszawa-Wrocław 1948.

[4] S. Lefschetz, Algebraic Topology, New York 1942.

[5] E. Marczewski and R. Sikorski, Measures in non-separable metric spaces, Coll. Math. 1 (1948), pp. 133-139.

[6] D. Montgomery, Non-separable Metric Spaces, Fund. Math. 25 (1935), pp. 527-533.

[7] A. H. Stone, Paracompactness and Product Spaces, Bull. Amer. Math. Soc. 54 (1948), pp. 977-982.

[8] J. Tukey, Convergence and Cniformity in Topology, Princeton 1940.

[9] N. Vedenisoff, Sur les fonctions continues dans les espaces topologiques, Fund. Math. 27 (1936), pp. 234-238.

Wrocław, May 1950.

\section{On Real-Valued Functions in Topological Spaces.}

By

\section{Ka tětov (Praha).}

The following theorem ${ }^{1}$ ) of H. Hahn is well-known: if $g$ and $h$ are real-valued functions in a metric space $P, g$ is upper semicontinuous ${ }^{2}$ ), $h$ is lower semicontinuous ${ }^{2}$ ), and $g(x) \leqslant h(x)$ for any $x \in P$, then there exists a continuous function $f$ such that $g(x) \leqslant f(x) \leqslant h(x)$ for erery $x \in P$. If $<$ is substituted for $\leqslant$, the theorem still holds. In his paper [4], J. Dieudonné has extended Hahn's theorem (with $\leqslant$ or $<$ ) to paracompact ${ }^{3}$ ) spaces. In the present note, it is shown that Hahn's theorem holds (i) with $\leqslant$, in arbitrary normal ${ }^{4}$ ) spaces (Theorem 1); (ii) with $<$, in a broad class (specified in Theorem 2) of normal spaces including paracompact, countably compact ${ }^{5}$ ) and perfectly normal ${ }^{6}$ ) ones (as a matter of fact, I do not know whether there exists any normal space not belonging to this class).

${ }^{1}$ ) See e. g. [5], 36. 2. 6 (numbers in brackets refer to the list at the end of the present note).

2) A real-valued function $g$ defined in a topological space $P$ is called upper semicontinuous if, for any $a \in P$ and any $c>g(a)$, there exists a neighbourhood $U$ of $a$ such that $c>g(x)$ whenever $x \in C$. Substituting $<$ instead of $>$, we obtain the definition of the lower semicontinuity.

3) A topological space $P$ is called paracompact if, for any open covering 5 of $P$, there exists an open covering $\mathfrak{S}$ which refines $(5$ (i. e. every $H \in \mathfrak{S}$ is contained in some $G \in(5)$ and is locally finite (i. e. such that every point has a neighbourhood intersecting only a finite number of sets $H \in \mathfrak{F}$ ). See J. Dieudonné's paper [4].

4) A topological space $P$ is ealled normal if any two disjoint closed sets possess disjoint neighbourhoods.

5) A topological space is called countably compact if every countable open covering contains a finite subcovering.

B) A normal space it called perfectly normal if every closech set can be represented as the intersection of countably many open sets. 
Theorem 3 of the present note concerns extensions of uniformly continuous functions defined in subsets of uniform spaces ${ }^{7}$ ). This theorem seems to be essentially known without having been explicitly stated as yet.

The proof of both Theorems 1 and 3 rests on a simple lemma concerning binary relations. Since Theorem 1 implies the classical Tietze-Urysohn Extension Theorem, we get, in this way, a direct proof of the Extension Theorem avoiding Urysohn's Lemma.

Notation. If $A, B$ are propositions, then $A \rightarrow B$ stands for "A implies $B$ ". „Function" always means a real-valued function.

Definitions. Let $R, T$ be sets, and let $\varrho, \tau$ be $n$-ary relations defined in $R$ and, respectively, in $T$. Then $R^{T}$ denotes the set of all transformations or the set $T$ into $R$, and $\varrho^{\tau}$ denotes then. $n$-ary relation in $R^{T}$ defined as follows: $\varrho^{\tau}\left(f_{1}, \ldots, f_{n}\right)$ if and only if $\tau\left(t_{1}, \ldots, t_{n}\right) \rightarrow \varrho\left(f_{1}\left(t_{1}\right), \ldots, f_{n}\left(t_{n}\right)\right)$.

We shall say that a binary relation $\varrho$ in $R$ possesses the Interpolation Property (cf. Birkhoff [1], p. 52) if, given finite sets $A \subset R_{\text {, }}$ $B C R$ such that ${ }^{8}$ ) $a \varrho b$ whenever $a \in A, b \in B$, there always exists $c \in R$ such that $a \varrho c, c \varrho b$ whenever $a \in A, b \in B$.

Lemma. Let a binary relation $\varrho$ in $R$ possess the Interpolation Property. Let $T$ be countable and let $\tau$ be a transitive irreflexive (i.e. such that tit never holds) relation in $T$. Then, for any $g \in R^{T}$ and

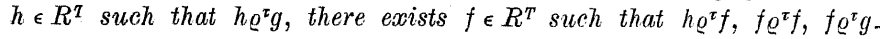

$\left.{ }^{7}\right)$ Let $P$ be a set and let $\mathcal{U}$ be a family of sets $U C P \times P$ such that (1) every set $U \in \mathcal{U}$ contains all $(x, x), x \in P$; (2) if $U \in \mathfrak{U}, U \subset V \subset P \times P$, then $V \in \mathcal{U}$; (3) if $U_{1} \in \mathfrak{U}, U_{2} \in \mathfrak{U}$, then $U_{1} U_{2} \in \mathfrak{U}$; (4) for any $U \in \mathfrak{U}$, there exists $V \in \mathfrak{U}$ such that $(z, x) \in \mathfrak{U}$ whenever $(x, y) \in \nabla,(y, z) \in \nabla$. Then we shall say that $\mathcal{U}$ is a uniformity in $P$; the set $P$ together with the uniformity $\mathfrak{U}$, will be called a uniform. space (see e.g. Bourbaki [2]). Example: a metric space $P$ with $\mathcal{u}$ consisting of all $U \subset P \times P$ containing, for some $\varepsilon>0$, all $(x, y)$ with $\rho(x, y)<\varepsilon$.

$A$ uniform space $P$ is always given the topology defined as follows: $x \in \bar{M}$ if and only if every $U \in \mathfrak{U}$, where $\mathfrak{U}$ denotes the uniformity of $P$, contains some $(x, y), y \in M$.

A real-valued function $f$ defined in a uniform space $P$ (with the uniformity $\mathfrak{U}$ ) is called uniformly continuous if, for any $\varepsilon>0$, there exists $U \in \mathfrak{U}$ such that $|f(x)-f(y)|<\varepsilon$ whenever $(x, y)^{-} \epsilon U$. This is, evidently a generalisation of the notion of uniform continuity in metric spaces.

2) If is a binary relation, then $a \rho b$ means, of course, that $a$ is in the re lation $e$ to $b$,
Remark. Evidently, $f \varrho^{\tau} f$ if and only if $f$ is a "homomorphism" with respect to $\tau, \varrho$, i. e. if $t_{1} \tau t_{2}$ implies $f\left(t_{1}\right) \varrho f\left(t_{2}\right)$.

Proof. Let all $t \in T$ be arranged in a sequence $\left\{t_{n}\right\}, t_{m} \neq t_{n}$ for $m \neq n$, and let $T_{n}(n=1,2, \ldots)$ denote the set of $t_{k}, k \leqslant n$. Suppose (which we evidently may for $n=1$ ) that

$\left(\mathrm{C}_{n}\right)$ if $t \tau t^{\prime}, t \in T_{n}, t^{\prime} \in T_{n}$, then $h(t) \varrho f\left(t^{\prime}\right), f(t) \varrho f\left(t^{\prime}\right), f(t) \varrho g\left(t^{\prime}\right)$.

Let $M$ denote the set of all $h(t)$ and $f(t)$ where $t \in T_{n}, t \tau t_{n+1}$, and let $N$ denote the set of all $f(t)$ and $g(t)$ where $t \in T_{n}, t_{n+1} \tau t$. Since $\tau$ is transitive, we have $x \varrho y$ whenerer $x \in M, y \in X$. Therefore, by the Interpolation Property, there exists $z \in R$ such that $x \in M \rightarrow x o z$, $y \in N \rightarrow z \varrho y$. Putting $f\left(t_{n+1}\right)=z$ we see at once that $\left(\mathrm{C}_{n+1}\right)$ holds true. The proof is now completed by an obvious induction.

Theorem 1. If $P$ is a normal space, $g$ and $h$ are functions in $P, g$ is upper semicontinuous, $h$ is lower semicontinuous, and $g(x) \leqslant h(x)$ for any $x \in P$, then there exists a continuous function $f$ in $P$ such that, for any $x \in P, g(x) \leqslant f(x) \leqslant h(x)$.

Proof. Let $R$ denote the collection of all $X \subset P$; if $X \in R$, $Y \in R$, put $X_{O} Y$ if and only if $\bar{X} \subset \operatorname{Int} X$. Let $\tau$ be the relation of (natural) order in the set $T$ of rational numbers (that is, $t \tau t^{\prime} \rightleftarrows t<t^{\prime}$ ). For any rational $t$, let $H(t)$ denote the set of $x \in P$ such that $h(x) \leqslant t$, and let $G(t)$ denote the set of $x \in P$ such that $g(x)<t$. It is easy to see that every $H(t)$ is closed, every $G(t)$ is open, and $t_{1}<t_{2} \rightarrow H\left(t_{1}\right) \subset G\left(t_{2}\right)$. Thus we have $G \in R^{T}, H \in R^{T}$, $\dot{H} \varrho^{\tau} G$. Since $\varrho$ has the Interpolation Property (this follows at once from the normality of $P$ ) there exists, br the above lemma, $F \in R^{T}$ such that $H Q^{\tau} F, F \varrho^{\tau} F, F \varrho^{\tau} G$, hence $\dot{H}\left(t_{1}\right) \subset \operatorname{Int} F\left(t_{2}\right), \overline{F\left(t_{1}\right)} \subset \operatorname{Int} F\left(t_{2}\right), \overline{F\left(t_{1}\right)} \subset \operatorname{Int} G\left(t_{2}\right)$ whenever $t_{i} \in T, t_{1}<t_{2}$. For any $x \in P$, let $f(x)$ be equal to the g.l.b. of numbers $t \in T$ such that $x \in F(t)$. Then $f$ is a real-valued function in $P$; for $\sum H(t)=P$, $\prod G(t)=0$, hence erery $x \in P$ lies in some $F(t)$ and in some $P-G(t)$, and therefore the values $f(x)= \pm \infty$ cannot occur. If $x \in F^{\prime}(t)$, then $x \in G\left(t^{\prime}\right)$ whenever $t^{\prime}>t$, and therefore $g(x) \leqslant t$; if $x$ non $\epsilon F(t)$, then $x$ non $\epsilon H\left(t^{\prime}\right)$ whenever $t^{\prime}<t$ and therefore $h(x) \geqslant t$. Hence $g(x) \leqslant f(x) \leqslant h(x)$ for every $x \in P$. If $t_{1}<f(x)<t_{2}, t_{i} \in T$, then it is easy to see that $x \in \operatorname{Int} F\left(t_{2}\right)-\overline{F\left(t_{1}\right)}$, and $y \in \operatorname{Int} \overline{F\left(t_{2}\right)}-F\left(t_{1}\right) \rightarrow t_{1} \leqslant f(y) \leqslant t_{2}$. Thus $f$ is eontinuous. 
From Theorem 1, it is easy to deduce ${ }^{9}$ ) the Tietze-Urysohn Extension Theorem: If $P$ is a normal space, $Q \subset P$ is closed, $f$ is continuous mapping of $P$ into an interval $\left.{ }^{10}\right) J$ of reals, then there exists a continuous mapping $F$ of $P$ into $J$ coinciding with $f$ in $Q$.

Remark. If $P$ is a non-normal completely regular ${ }^{11}$ ) space, then it may happen that, for some closed set $Q \subset P$, every bounded continuous function in $Q$ has an extension ${ }^{12}$ ) over $P$ whereas no unbounded continuous function in $Q$ has such an extension.

Example. Let $E$ be the space of real numbers and let $Q \subset E$ be the set of all integers. Let $\beta E$ denote the Cech (bi)compactification $\left.{ }^{13}\right)$ of $E$ and put $P=\beta E-(\bar{Q}-Q)$, where $\bar{Q}$ denotes, of course, the closure of $Q$ in $\beta E$. Since $E$ is normal, every bounded continuous function in $Q$ admits of an extension over $E$, hence over $P$. Now let $f$ be an unbounded continuous function in $Q$ and suppose that there exists a continuous function $F$ in $P$ such that $x \in Q \rightarrow F(x)=f(x)$. It is easy to see that there exists a closed (in $E$ ) set $A \subset E-Q$ such that $F(A)$ is not bounded. Since $\bar{Q}$ and $\bar{A}$ (closures in $\beta E$ ) are disjoint, the closure of $A$ in $P$ is equal to $\bar{A}$, hence compact. Thus $F(A)$ is bounded and we have a contradiction.

Theorem 2. If $P$ is normal, then the following conditions are equivalent:

(a) if $g, h$ are functions in $P, g$ is upper semicontinuous, $h$ is lower semicontinuous and $g(x)<h(x)$ for every $x \in P$, then there cxists a continuous function $f$ in $P$ such that, for any $x \in P, g(x)<f(x)<h(x)$;

(b) every countable open covering of $P$ has a locally finite refinement;

9) In a wèll-known way: if $I=[\alpha, \beta]$ is closed, put $\varphi(x)=\psi(x)=f(x)$ if $x \in Q$ and $\varphi(x)=\alpha, \psi(x)=\beta$ if $x \in P-Q$. Then $\varphi(x) \leqslant \psi(x), \varphi$ is upper semicontinuous, $\psi$ is lower semicontinuous. Hence there exists a continuous function $F$ in $P$ with $\varphi(x) \leqslant F(x) \leqslant \psi(x)$, for any $x \in P$. Clearly, $x \in Q \rightarrow F(x)=f(x)$. For the case of a nonclosed interval $I$ see e. g. Bourbaki [3], p. 65 .

${ }^{10}$ ) Any interval, closed or not, bounded or unbounded.

$\left.{ }^{11}\right)$ A topological space $P$ is called completely regular, if, for any closed $M C P$ and any $x \in P-M$, there exists a continuous function $f$ in $P$ such that $f(x)=1$ and $y \in M \rightarrow f(y)=0$.

$\left.{ }^{12}\right)$ This means: there exists a continuous function $F$ in $P$ such that $x \in Q \rightarrow F(x)=f(x)$.

${ }^{13}$ ) If $S$ is a completely regular space, then there exists an essentially unique compact (=bicompact) space, denoted by $\beta S$ and called the Cech compactification of $S$, such that $S \subset \beta S, \bar{S}=\beta S$ and every bounded continuous function in $S$ has an extension over $\beta S$. (c) every countable open covering of $P$ has a point-finite ${ }^{14}$ ) refinement;

(d) every countable open covering is shrinkable ${ }^{15}$ );

(e) if $F_{n} \subset P$ are closed, $F_{n} \subset F_{n+1}(n=1,2, \ldots), \prod_{n=1}^{\infty} F_{n}=0$, then there exist open sets $G_{n} \supset F_{n}$ such that $\prod_{n=1}^{\infty} G_{n}=0$.

Proof. I. If (a) holds, let $G_{n}$ be open, $\sum_{n=1}^{\infty} G_{n}=P$. Put

and put

$$
U_{n}=G_{1}+\ldots+G_{n} \quad(n=1,2, \ldots)
$$

$$
h(x)=\left\{\begin{array}{ll}
1 & \text { if } x \in U_{1}, \\
n^{-1} & \text { if } x \in U_{n}-U_{n-1}
\end{array} \quad(n=2,3, \ldots) .\right.
$$

Since $h$ is clearly a lower semicontinuous function, there exists (for we can put $g(x)=0$, for any $x$, and make use of the property (a)) a positive continuous function $f$ in $P$ such that $x \in U_{1} \rightarrow f(x)<1$,

$$
r \in U_{n}-U_{n-1} \rightarrow f(x)<n^{-1} \quad(n=2,3, \ldots) .
$$

Let $I_{k}(k=1,2, \ldots)$ denote the open interval with endpoints $(k+2)^{-1}, k^{-1}$ and put $H_{k}=f^{-1}\left(I_{k}\right)$. Clearly, $H_{k} \subset U_{k+1}(k=1,2, \ldots)$, $\sum_{k=1}^{\infty} H_{k}=P$, and every $x \in P$ has a neighbourhood intersecting two sets $H_{k}$ at most. It is easy to show that the collection of all nonroid sets $H_{k} G_{l}, l \leqslant k+1$, is a locally finite open covering of $P$ which refines $\left\{G_{n}\right\}$. Thus (a) implies (b).

II. Evidently, (b) implies (c).

III. If (c) holds, let $\left\{G_{n}\right\} \quad(n=1,2, \ldots)$ be an open covering of $P$. Let $\left\{H_{\nu}\right\}, v$ running orer an arbitrary given set of indices, be a point-finite refinement of $\left\{G_{n}\right\}$. For any $v$, choose $m=m(v)$ such that $H_{v} \subset G_{m}$. Let $U_{n}(n=1,2, \ldots)$ denote the sum of all $H_{v}$ such that $m(v)=n$. Then $U_{n} \subset G_{n}(n=1,2, \ldots),\left\{U_{n}\right\}$ is point-finite. Now apply the following well-known (see e. g. Lefschetz [5], p. 26) theorem: every point-finite covering of a normal space is shrinkable.

14) A covering $\mathfrak{A}$ of a space $P$ is called point-finite if erery $x \in P$ belongs to a finite number of sets $\mathcal{A} \in \mathfrak{A}$.

15) If $\left\{G_{v}\right\}, v$ running orer an arbitrary given set of indices, is an open covering of a space $P$, then we shall say that $\left\{G_{\nu}\right\}$ is shrinkable if there exist closed sets $F_{\nu} \subset G_{\nu}$ such that $\sum_{y} F_{\nu}=P$. Cf. Lefschetz [5], p. 26 . 
IV. If (d) holds, let $F_{n}$ be closed, $F_{n} \supset F_{n+1}, \prod_{n=1}^{\infty} F_{n}=0$. Put $H_{n}=P-F_{n}$. Then $\sum_{n=1}^{\infty} H_{n}=P$ and therefore there exist closed sets $A_{n} \subset H_{n}$ such that $\sum_{n=1}^{\infty} A_{n}=P$. Put $G_{n}=P-A_{n}$. Then $G_{n} \supset F_{n}, \prod_{n=1}^{\infty} G_{n}=0$.

T. If (e) holds, let $g$ and $h$ be functions in $P$; suppose that $g$ is upper semicontinuous, $h$ is lower semicontinuous, $g(x)<h(x)$ for all $x \in P$. Let $F_{n}(n=1,2, \ldots)$ denote the set of $x \in P$ such that $h(x)-g(x) \leqslant 3^{-n+1}$. Clearly, $F_{n} \supset F_{n+1}, \prod_{n=1}^{\infty} F_{n}=0$. Therefore, there exist open sets $G_{n} \supset F_{n}$ such that $\prod_{n=1}^{\infty} G_{n}=0$. Since $P$ is normal, there exist continuous functions $\varphi_{n}$ in $P(n=1,2, \ldots)$ such that we always have $0 \leqslant \varphi_{n}(x) \leqslant 1, \varphi_{n}(x)=0$ if $x \in F_{n}, \varphi_{n}(x)=1$ if $x \in P-G_{n}$. For any $x \in P$, put $\varphi(x)=\sum_{n=1}^{\infty} 3^{-n} \varphi_{n}(x)$. Then $\varphi$ is a continuous function in $P$. Clearly we have $0<\varphi(x) \leqslant \frac{1}{2}$, for any $x \in P$, and $\varphi(x) \leqslant \frac{1}{2} 3^{-n}$ for $x \in F_{n}$. Since every $x \in P$ lies either in $P-F_{1}$ or in some $F_{n}-F_{n+1}$, we get at once $2 \varphi(x)<h(x)-g(x)$, for any $x \in P$. Putting $g_{1}=g+\varphi$, $h_{1}=h-\varphi$ and applying Theorem 1 to $y_{1}$ and $h_{1}$, we see that (e) implies (a). This completes the proof.

Remarks. $1^{0} \mathrm{I}$ do not know whether there exists any normal space which does not possess properties (a)-(e).

$2^{0} \mathrm{It}$ is clear that every paracompact or countably compact space has property (b) and every perfectly normal space has property (e). Hence the class of normal spaces possessing properties (a)-(e) includes paracompact, countably compact and perfectly normal ones.

We shall now consider uniformly continuous functions in uniform spaces.

Theorem 3. Let $P$ be a uniform space and let $f$ be a bounded. uniformly continuous function in a subspace $Q \subset P$. Then there exists a bounded uniformly continuous function $F$ in $P$ which coincides with $f$ in $Q$.

Proof. Let $R$ denote the collection of all $X \subset P$. If $X \in R$, $Y \in R$, put $X \varrho Y$ if and only if there exists $U \in \mathfrak{U}$ (where $\mathfrak{U}$ denotes, of course, the uniformity of the space $P$ ) such that $x \in X$, $(x, y) \in U \rightarrow y \in Y$. It is easy to see (cf. footnote $\bar{\tau}$, property (4)) that the relation $\varrho$ has the Interpolation Property. Let $T$ denote the set of rational numbers; if $t_{1} \in T, t_{2} \in T$ put $t_{1} \tau t_{2} \rightleftarrows t_{1}<t_{2}$. Let $a$ and $\beta$ denote respectively the g. l. b. and the 1 . u. b. of numbers $f(x), x \in Q$. If $t \in T, \alpha \leqslant t \leqslant \beta$, let $A(t)$ denote the set of points $x \in Q$ such that $f(x) \leqslant t$ and put $B(t)=A(t)+(P-Q)$. If $t \in T, t<a$, put $A(t)=B(t)=0$; if $t \in T, t>\beta$, put $A(t)=B(t)=P$. It is easy to see that, for $t_{1} \in T, t_{2} \in T$, $t_{1}<t_{2}$ implies $A\left(t_{1}\right) \varrho B\left(t_{2}\right)$. Thus we have $A \varrho^{\tau} B$ and therefore, by the lemma on binary relations, there exists $C \epsilon R^{T}$ such that $A Q^{\tau} C$, $C \varrho^{\tau} C, C \varrho^{\tau} B$, that is $A\left(t_{1}\right) \varrho C\left(t_{2}\right), C\left(t_{1}\right) \varrho C\left(t_{2}\right), C\left(t_{1}\right) \varrho B\left(t_{2}\right)$ whenever $t_{i} \in T, t_{1}<t_{2}$.

For any $x \in P$, let $F(x)$ be equal to the g. l. b. of numbers $t \in T$ such that $x \in C(t)$. If $x \in Q, t_{1}<f(x)<t_{2}$, where $t_{i} \in T$, then clearly $x \in \mathcal{A}\left(t_{2}\right)-B\left(t_{1}\right)$ and therefore $x \in C\left(t_{2}^{\prime}\right)-C\left(t_{1}^{\prime}\right)$ whenever $t_{i}^{\prime} \epsilon T$, $t_{1}^{\prime}<t_{1}, t_{2}<t_{2}^{\prime}$; hence $t_{1} \leqslant F(x) \leqslant t_{2}$. Therefore, $x \in Q \rightarrow F(x)=f(x)$. Clearly, $x \in P \rightarrow \alpha \leqslant F(x) \leqslant \beta$.

It remains to prove that $F$ is uniformly continuous. Given $\varepsilon>0$, choose numberr $t_{k} \in T(k=0,1, \ldots, n+1)$ such that $0<t_{k+1}-t_{k}<\frac{1}{2} \varepsilon$, $t_{0} \leqslant \alpha, \beta \leqslant t_{n+1}$. Since $C\left(t_{k}\right) \underline{Q} C\left(t_{k+1}\right)$, there exist sets $U_{k} \in \mathfrak{U}(k=0,1, \ldots, n)$ such that $y \in C\left(t_{k+1}\right)$ whenerer $x \in C\left(t_{k}\right),(x, y) \in U_{k}$. Put $U=\prod_{k=0}^{n} U_{k}$. Then $U \in \mathfrak{U}$ (ef. footnote $\bar{\tau}$, property (3)). Clearly we have $F(y) \leqslant t_{k+1}$ whenever $F(x)<t_{k},(x, y) \in U$; therefore, $|F(x)-F(y)| \leqslant \varepsilon$ whenever $(x, y) \in U$. This completes the proof.

\section{References.}

[1] G. Birkhoff, Lattice Theory, revised edition, New York 1948.

[2] N. Bourbaki, Eléments de mathématiques, Les structures fondamentales de l'analyse, Livre III, Topologie générale, Chap. I et II, Paris 1940.

[3] -, Eléments de mathématique, Les structures fondamentales de l'analyse, Livre III, Topologie générale, Chap. IX, Paris 1948.

[4] J. Dieudonné, Cue généralisation des espaces compacts, J. Math. Pures et Appliquées (9) 23, (1944), pp. 65-76.

[5] H. Hahn, Reelle Funktionen I, Wien 1932.

[6] S. Lefschetz, Algebraic Topology, New York 1942. 